Proc. Indian Acad. Sci. (Earth Planet. Sci.), Vol. 95, No. 3, November 1986, pp. 331-341.

(C) Printed in India.

\title{
Magnetic anomalies over thin plates and their analysis
}

\author{
H V RAMBABU and G D J SIVAKUMAR SINHA \\ National Geophysical Research Institute, Hyderabad 500007 , India
}

MS received 10 July 1985; revised 7 January 1986

\begin{abstract}
The magnetic anomaly over a two-dimensional thin horizontal plate is similar to the first horizontal derivative of the magnetic anomaly over a thick dipping dike of infinite depth extent but with a different direction of magnetization. Hence, the magnetic anomalies of thin plates may be integrated along the profile and the pseudomagnetic potential anomaly thus obtained may be interpreted using any standard method of interpreting dike anomalies. Expressions for the Fourier amplitude and phase spectra of the magnetic anomaly over a thin plate are also derived and procedures to evaluate the parameters of the plate from the spectra are formulated.
\end{abstract}

Keywords. Thin plate; magnetic interpretation; Fourier transform; pseudo-magnetic potential.

\section{Introduction}

Quantitative interpretation of isolated magnetic anomalies is usually carried out by assuming the sources to be of simple geometric shapes such as dikes, sheets, steps, etc. The anomalies caused by undulations in the basement surface are often attributed to a prism model, but the thin plate model appears to be a better approximation due to the small thickness-to-depth ratio of the suprabasement feature. The authors are not aware of any published work relating to the interpretation of magnetic anomalies over thin plates except the work of Telford et al (1976) and Nettleton (1976), which is only elementary in nature.

In the present work, the mathematical expression for induced magnetic anomalies over thin plates is derived. Two methods for calculating the depth, width and susceptibility of the plate are suggested. In the first method, the anomaly is integrated with distance to obtain a profile of pseudo-magnetic potential denoted by $P(x)$. This resembles the magnetic anomaly of a dike with a different direction of magnetization and hence, any standard method of dike interpretation may be used to obtain the body parameters. The curve matching technique of Gay (1963), the method of characteristic curves of Koulomzine et al (1970) or Atchuta Rao and Ram Babu (1981), the computer method of Rao et al (1973), the spectral method of Bhimasankaram et al (1977) or Rao et al (1978) etc., are a few methods for interpreting magnetic anomalies over thick dikes. In the second method, the Fourier spectra of the anomaly are analyzed to evaluate the parameters of the plate.

\section{Theory}

2.1 General expression for the magnetic anomaly over a thin plate

In formulating the expression for the magnetic anomaly due to a thin plate (figure 


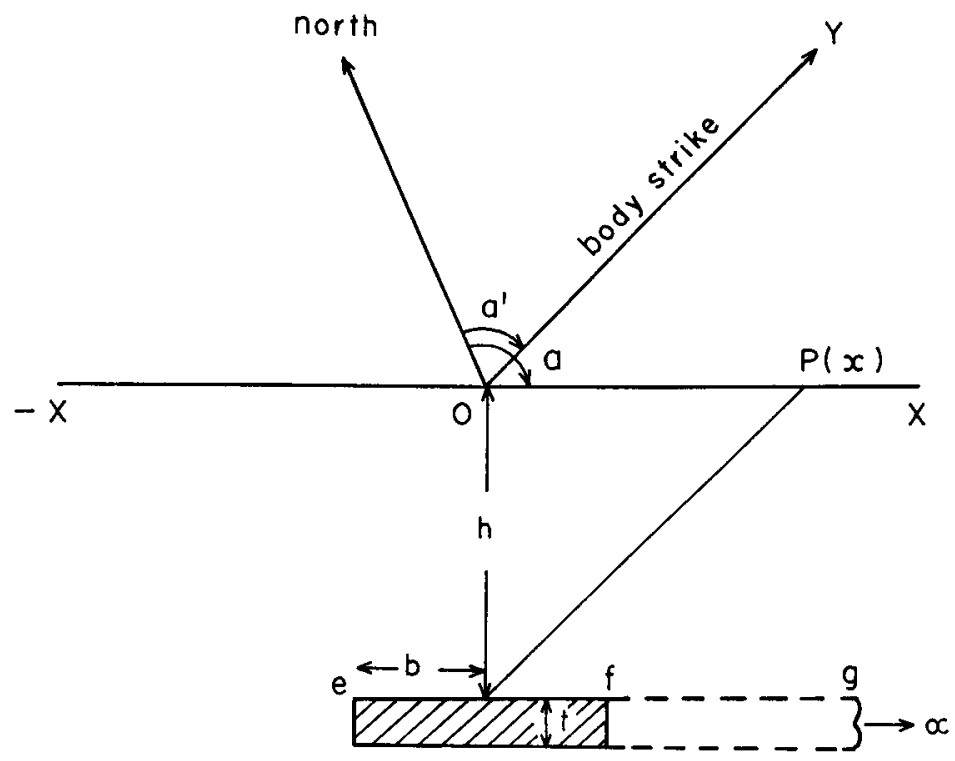

Figure 1. Geometry of a two-dimensional thin plate.

1), the following notation has been used. In an $X O Y$ cartesian coordinate system (figure 1), the $Y$-axis is chosen along the strike of the body. The magnetic profile is along the $X$-axis, making an angle $a$ with the magnetic north, $a^{\prime}$ is the strike azimuth, $i$ is the inclination of the earth's magnetic field of intensity $T, k$ is the susceptibility contrast of the body to its surroundings; $I$ is the effective inclination in a plane perpendicular to the strike of the plate and is given as follows:

$$
\tan I=\tan i / \cos a .
$$

The magnetic anomaly of a thin horizontal plate $e f$ of thickness $t$ and width $2 b$ buried at a depth $h$ may by found by working out the difference in the magnetic effects of two suitably disposed semi-infinite sheets $e g$ and $f g$.

The magnetic effect $F_{e g}(x)$ due to the semi-infinite sheet $e g$ is given by the following equation (modified after Gay, 1963):

$$
F_{e g}(x)=C_{p} \frac{(x+b) \sin Q_{p}+h \cos Q_{p}}{(x+b)^{2}+h^{2}},
$$

where $x$ is the distance of the point of observation from the origin $O, C_{p}$ and $Q_{p}$ are the amplitude coefficient and the index parameter respectively whose equivalents are given in table 1 for the three cases $(\Delta T, \Delta Z$, and $\Delta H)$ of $F(x)$.

Similarly, the magnetic effect $F_{f g}(x)$ due to the sheet $f g$ may be written as follows:

$$
F_{f g}(x)=C_{p} \frac{(x-b) \sin Q_{p}+h \cos Q_{p}}{(x-b)^{2}+h^{2}},
$$


Table 1. Equivalents of the amplitude coefficient $(C)$ and index parameter $(Q)$ for the plate and the dike.

\begin{tabular}{llc}
\hline $\begin{array}{l}\text { Anomaly } \\
(F)\end{array}$ & $\begin{array}{l}\text { Amplitude } \\
\text { coefficient }\end{array}$ & $\begin{array}{c}\text { Index } \\
\text { parameter }\end{array}$ \\
\hline (a) for thin plate & $C_{p}$ & $Q_{p}$ \\
$T$ & $2 k T t B^{2}$ & $2 I_{p}-90$ \\
$Z$ & $2 k T t B$ & $I_{p}$ \\
$H$ & $2 k T t B \cos a$ & $I_{p}-90$ \\
(b) for thick dike & $C_{d}$ & $Q_{d}$ \\
$T$ & $2 k T B^{2} \sin (\delta)$ & $2 I_{d}-\delta 90$ \\
$Z$ & $2 k T B \sin (\delta)$ & $I_{d}-\delta$ \\
$H$ & $2 k T B \sin (\delta) \cos a$ & $I_{d}-\delta-90$ \\
\hline
\end{tabular}

The general expression for the magnetic anomaly $F_{p}(x)$ over the plate ef may be written as follows:

$$
F_{p}(x)=C_{p}\left[\frac{(x+b) \sin Q_{p}+h \cos Q_{p}}{(x+b)^{2}+h^{2}}-\frac{(x-b) \sin Q_{p}+h \cos Q_{p}}{(x-b)^{2}+h^{2}}\right]
$$

\subsection{Type curves of plate anomalies}

Equation (3) gives the magnetic anomaly in either the total $(\Delta T)$, vertical $(\Delta Z)$, or horizontal $(\Delta H)$, field at any point $P(x)$ on a line perpendicular to the strike of a two-dimensional thin horizontal plate of width $2 b$ situated at a depth $h$ below the line of observation. It may be noted from (3) that the amplitude of $F_{p}(x)$ depends on $C_{p}$ whereas its shape depends on $Q_{p}, b$ and $h$. A few sets of type curves over the plate are presented in figure 2 .

The $\Delta T$ anomaly over a thin plate $(b / h=2)$, striking east-west is shown in figure $2 \mathrm{a}$ for different inclinations of the earth's field. It may be noted from figure $2 \mathrm{a}$ that at equator $\left(i=0^{\circ}\right)$, the magnetic anomaly is a symmetric low flanked on either side by a small high of equal amplitude. At the pole $\left(i=90^{\circ}\right)$, the curve is a mirror image along $x$-axis of the $\Delta T$ curve for $i=0^{\circ}$. In intermediate latitudes $\left(i=30^{\circ}\right.$ and $60^{\circ}$ ), the curve consists of a low towards the northern end.

The $\Delta T$ anomaly over a plate $\left(b / h=2 ; i=30^{\circ}\right)$ for various strike directions (figure $2 \mathrm{~b}$ ) shows that when the strike of the plate is along north-south $\left(=0^{\circ}\right)$, the anomaly is a symmetric high flanked on either side by a small low. As the strike changes from north-south to east-west, the northern low enhances while the southern low diminishes.

Variations in the $\Delta T$ anomaly over a plate when the width of the plate changes may be noted from figure $2 \mathrm{c}$ when $a=0^{\circ}$ and $i=30^{\circ}$. It is seen that the anomaly stretches horizontally as the width increases and for half-widths equal to or greater than 3 depth units, the low and the high occur approximately over the edges of the plate.

\subsection{Relation between magnetic anomalies over thin plates and thick dikes}

The magnetic anomaly over a thick dipping dike of the same width as the thin plate may be written as follows (after Gay 1963): 

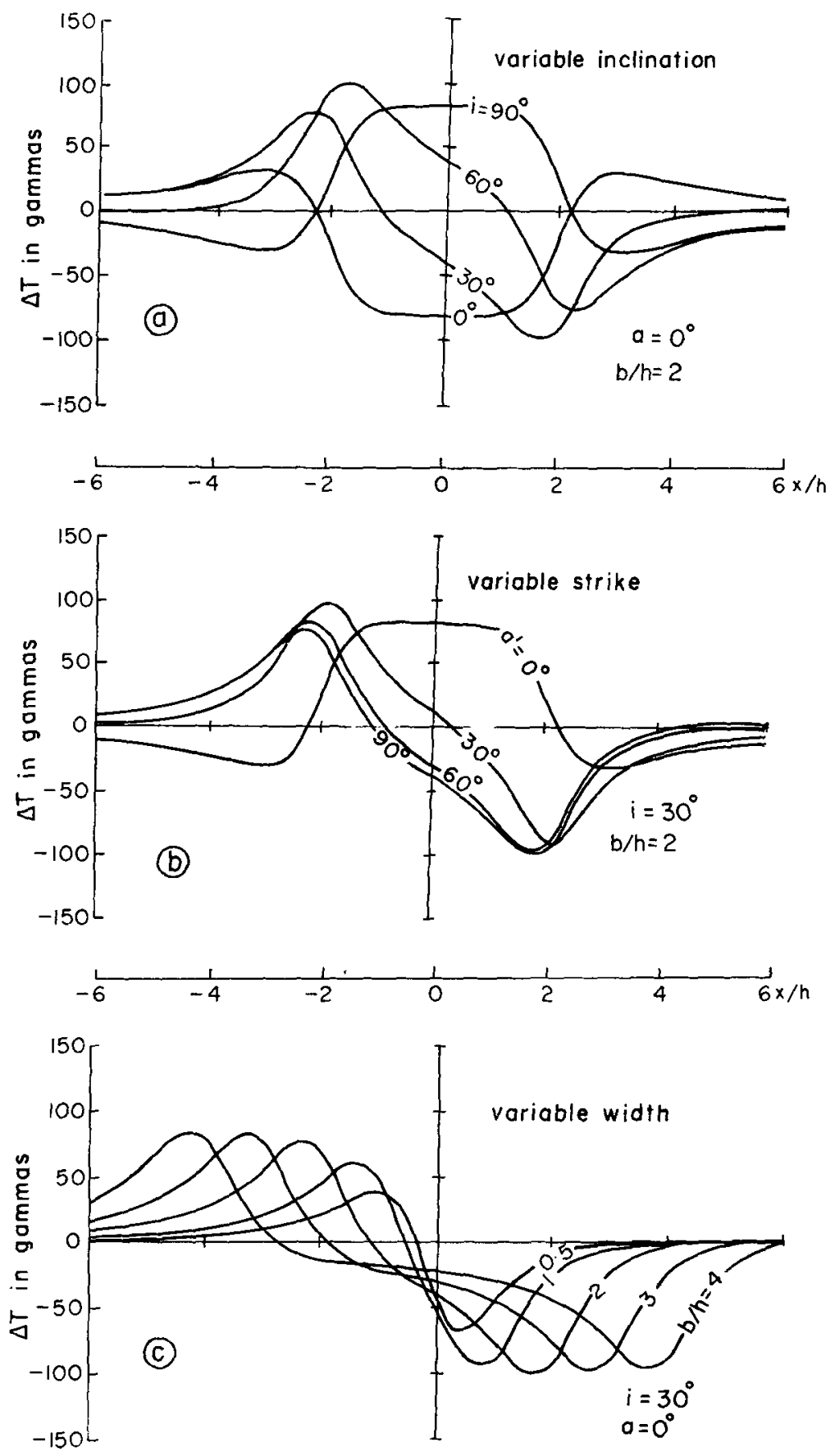

Figure 2. Model magnetic anomalies over a two-dimensional thin plate; $(a)$ variable inclination, $(b)$ variable strike, and $(c)$ variable width. 


$$
\begin{aligned}
F_{d}(x)= & C_{d}\left[\cos Q_{d}\left(\arctan \frac{x+b}{h}-\arctan \frac{x-b}{h}\right)\right. \\
& \left.+0.5 \sin Q_{d} \ln \frac{(x+b)^{2}+h^{2}}{(x-b)^{2}+h^{2}}\right]
\end{aligned}
$$

where the cofficients $C_{d}$ and $Q_{d}$ are defined in table 1 in terms of the inclination $(i)$, azimuth $(a)$ of the profile and $\operatorname{dip}(\delta)$ of the dike etc. The horizontal deritive $F_{d x}(x)$ may now be written as follows:

$$
\begin{aligned}
F_{d x}(x) & =C_{d}\left[\cos Q_{d}\left(\frac{h}{(x+b)^{2}+h^{2}}-\frac{h}{(x-b)^{2}+h^{2}}\right)\right. \\
& \left.+\sin Q_{d}\left(\frac{(x+b)}{(x+b)^{2}+h^{2}}-\frac{(x-b)}{(x-b)^{2}+h^{2}}\right)\right]
\end{aligned}
$$

when $Q_{p}$ and $Q_{d}$ are identical, the following identity may be written from (3) and (5).

$$
\frac{F_{p}(x)}{C_{p}} \equiv \frac{F_{d x}(x)}{C_{d}} .
$$

If the effective dips of magnetization of the dike and the plate are denoted as $I_{d}$ and $I_{p}$ respectively, then under the condition $Q_{p}=Q_{d}, I_{p}=I_{d}-\delta / 2$ for $\Delta T$ anomaly, and $I_{p}=I_{d}-\delta$ for $\Delta Z$ and $\Delta H$ anomalies.

\subsection{Analysis of plate anomalies}

The magnetic anomaly $\left[F_{p}(x)\right]$ over a plate may now be computed using (3) when the parameters of the plate are known. But, in practice, it is necessary to find the parameters of the plate when $F_{p}(x)$ is known. We suggest the following two methods for finding the parameters of the plate from $F_{p}(x)$.

\subsection{The pseudo-magnetic potential method}

Integrating $F_{p}(x)$ with respect to $x$, we obtain the expression for $P(x)$, the pseudo-magnetic potential anomaly as follows:

$$
\begin{aligned}
P(x)= & \int_{-\infty}^{x} F_{p}(x) \mathrm{d} x \\
= & C_{p}\left[\cos Q_{p} \arctan \left(\frac{x+b}{h}-\arctan \frac{x-b}{h}\right)\right. \\
& \left.+0 \cdot 5 \sin Q_{p} \ln \frac{(x+b)^{2}+h^{2}}{(x-b)^{2}+h^{2}}\right] .
\end{aligned}
$$


Again under the condition $Q_{p}=Q_{d}$ the following identity may be written from equations (4) and (7):

$$
\frac{P(x)}{C_{p}} \equiv \frac{F_{d}(x)}{C_{d}}
$$

It may be inferred from (8) that the function $P(x)$ is identical to $F_{d}(x)$ (except for the amplitude factor). Hence, any technique available for interpreting dike anomalies can be used to interpret the plate anomalies by making use of the function $P(x)$. In the example presented here, the curve matching technique of Gay (1963) is adopted.

\subsection{The spectral method}

The Fourier transform $\bar{F}(w)$ of $F_{p}(x)$ may be written as follows:

$$
\bar{F}(w)=\int_{-\infty}^{\infty} F_{p}(x) \exp (-j w x) \mathrm{d} x .
$$

Using the following transform pairs (Erdelyii 1954)

and

$$
h /\left(x^{2}+h^{2}\right) \rightarrow \pi \exp (-w h)
$$

$$
x /\left(x^{2}+h^{2}\right) \rightarrow \pi j \exp (-w h),
$$

and the Fourier shift theorem, the final expression for $\bar{F}(w)$ the Fourier transform of $F_{p}(x)$ may be written as follows:

$$
\bar{F}(w)=2 j \pi C_{p} \exp (-w h) \exp \left(j Q_{p}\right) \sin w b .
$$

Since, for field data, the origin is unknown, the distances are measured from a reference point which is at a distance $D$ from the origin. In this case, (10) may be rewritten as follows:

$$
\bar{F}(w)=2 \pi j C_{p} \exp (-w h) \exp \left[j\left(Q_{p}-w D\right)\right] \sin w b .
$$

The amplitude $A(w)$ and phase $\phi(w)$ of $F(w)$ may be written from (11) as follows:

$$
A(w)=2 \pi C_{p} \exp (-w h) \sin w b
$$

and

$$
\Phi(w)=\pi / 2+w / D-Q_{p} .
$$

It may be noted from (12) that the amplitude $A(w)$ is an exponentially decaying sinusoid (figure 3). $A(w)=0$ whenever $w b$ is equal to $n \pi(n=0,1,2, \ldots)$. If $A(w)=0$ successively at two frequencies $w_{1}$ and $w_{2}$, then 


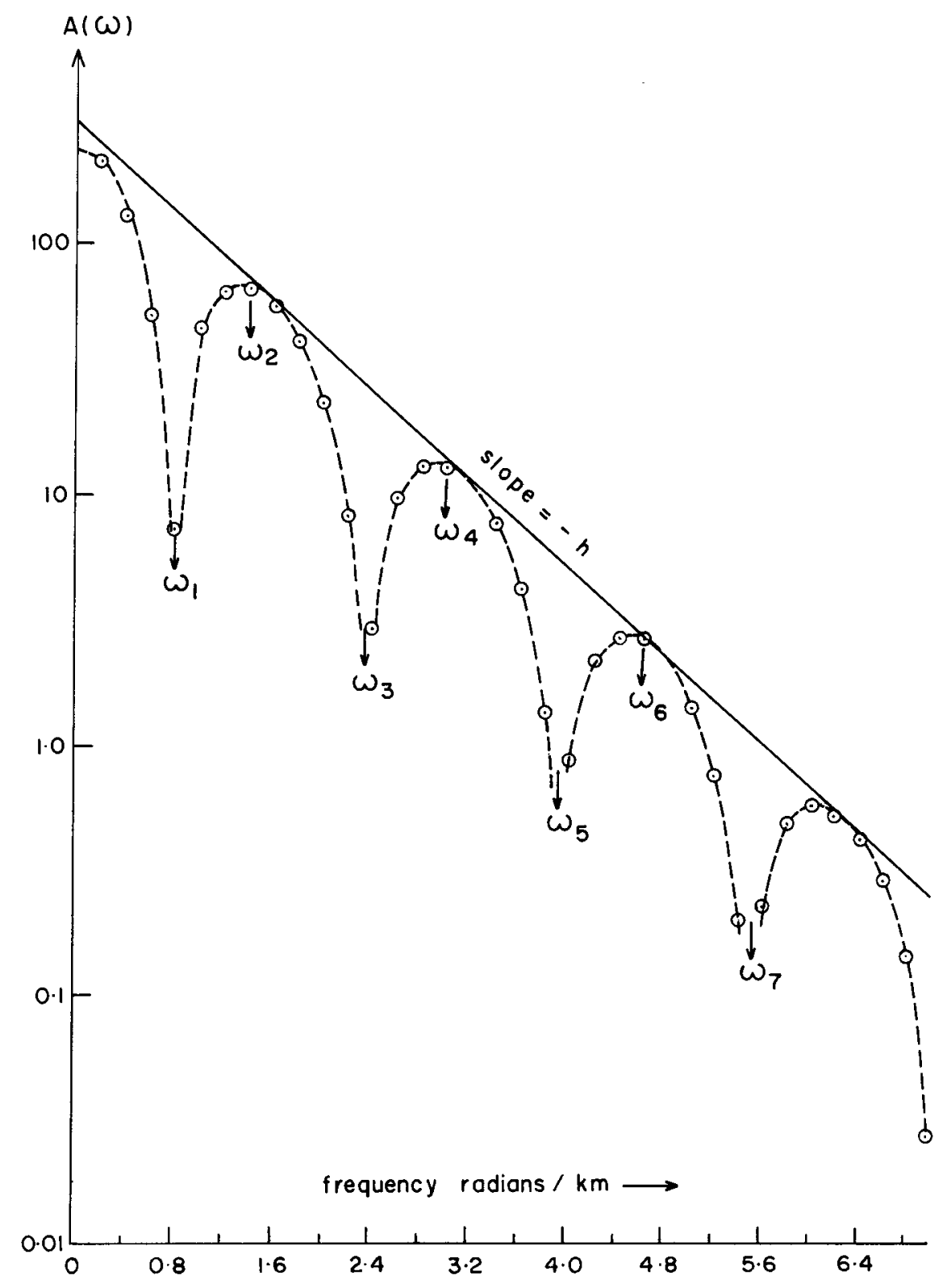

Figure 3. Theoretical Fourier amplitude $[A(w)]$ spectrum of the magnetic anomaly over a thin plate.

$w_{1} b=n \pi$, and $w_{2} b=(n+1) \pi$

so that

$$
b=\pi /\left(w_{2}-w_{1}\right)
$$


Defining the modified amplitude spectrum $A_{l}(w)=A(w) / \sin (w b)$, equation (12) can be modified as follows:

$$
A_{1}(w)=2 \pi C_{p} \exp (-w h)
$$

A plot of $\ln A_{1}(w)$ versus $w$ will be a straight line whose slope and intercept are equal to $-h$ and $\ln \left(2 \pi C_{p}\right)$ respectively from which $h$ and $C_{p}$ may be calculated. After finding $C_{p}$, the thickness $(t)$ of the plate may be evaluated using table 1 by assuming the susceptibility contrast $(k)$ or vice versa.

The phase $[\phi(w)]$ spectrum will be a straight line whose slope is equal to $D$, the distance of the origin from the starting point of the profile. The intercept of this line is equal to $\left(\frac{1}{2} \pi-Q_{p}\right)$.

Thus, using the properties of the Fourier spectra, it is possible to evaluate the parameters of the plate.

\section{Example}

The aeromagnetic anomaly, $10 \mathrm{~km}$ south of Tezpur, (Assam state) in north eastern India is shown in figure $4 \mathrm{a}$. The observations were made along a line making an angle of $45^{\circ}$ from the magnetic north towards east at an elevation of $1.36 \mathrm{~km}$ above sea level. The inclination, declination and intensity of the earth's magnetic field are $39^{\circ} \mathrm{N}, 1 / 3^{\circ} \mathrm{W}$ and $47500 \mathrm{nT}$ respectively. The area is covered by Brahmaputra alluvium overlying the tertiary sediments occurring above the archaean basement. The sediments being non-magnetic cannot produce the observed magnetic anomaly. Hence, this anomaly might be due to the basement topography which may be approximated to a thin plate model. Using the two methods presented in this paper, this anomaly has been interpreted in the following way.

\subsection{The pseudo-magnetic potential method}

The magnetic anomaly was integrated by successive addition to obtain $P(x)$. The function $P(x)$ is normalized to the same scale ( $2 \cdot 5$ inches) as the theoretical curves for thick dikes (Gay 1963) and compared by changing the distance scale suitably to obtain a good fit with one of the theoretical curves. Since in this case, $i=39^{\circ}$, $a=45^{\circ}$ and $I=50^{\circ}$ hence, $Q_{p}=10^{\circ}$. The $P(x)$ curve should match with one of the dike curves whose $Q_{d}=10^{\circ}$. It may be seen from figure $4 \mathrm{~b}$ that the $P(x)$ curve fits well with the dike curve whose $Q_{d}$ and $R$ (the width-to-depth ratio) values are $10^{\circ}$ and 5 (extrapolated curve) respectively. The parameters of the plate obtained in this way are presented in table 2 .

\subsection{The spectral method}

The $\Delta T$ anomaly of length $31.5 \mathrm{~km}$ is digitized at $0.5 \mathrm{~km}$ interval and its discrete Fourier transform is computed. From the amplitude $A(w)$ spectrum (figure 5) of the Fourier transform, the depth, width and susceptibility (for $t=0.5 \mathrm{~km}$ ) of the plate are evaluated and are also shown in table 2 . The low susceptibility value of 0.006 c.g.s. is characteristic of granitic basement. It may be inferred from our 


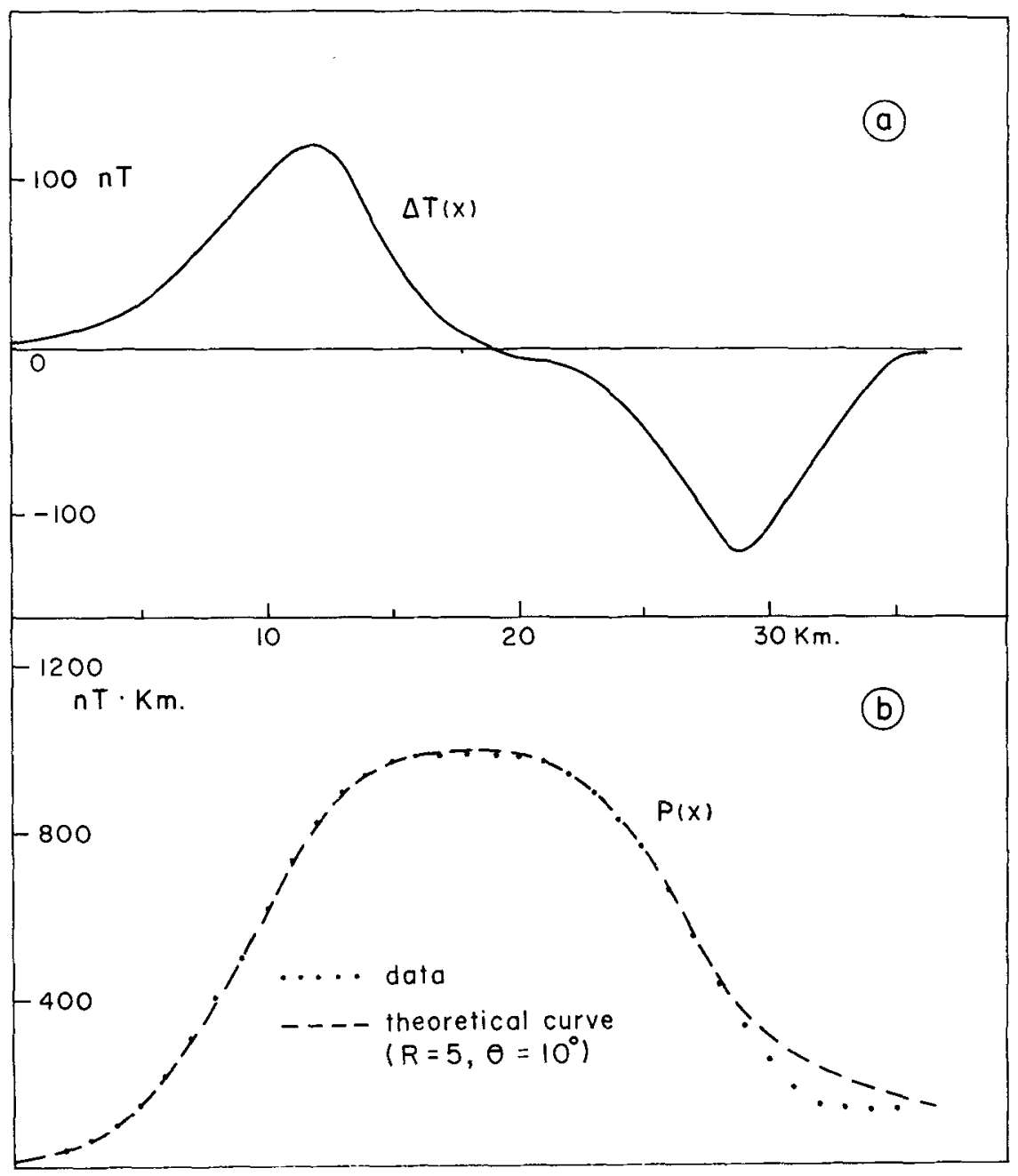

Figure 4. (a) An aeromagnetic anomaly, near Tezpur (Assam state, India), (b) interpretation of $P(x)$ curve using the standard curves of Gay (1963).

Table 2. Results of interpretation of the aeromagnetic anomaly near Tezpur, using the thin plate model.

\begin{tabular}{lcc}
\hline & $\begin{array}{c}\text { The pseudo magnetic } \\
\text { potential method }\end{array}$ & $\begin{array}{c}\text { Spectral } \\
\text { method }\end{array}$ \\
\hline Parameter & 3.36 & 3.90 \\
$\begin{array}{l}\text { Depth }(h \text { in } \mathrm{km} \text { ) to top } \\
\text { (below flight level) }\end{array}$ & & \\
Depth ( $h$ in $\mathrm{km}$.) to top & 2.00 & 2.54 \\
$\quad$ Width (2b in km) & 16.65 & 15.80 \\
Susceptbility $(k)(\mathrm{c} . \mathrm{g} . \mathrm{s})$. & 0.006 & 0.006 \\
\hline
\end{tabular}




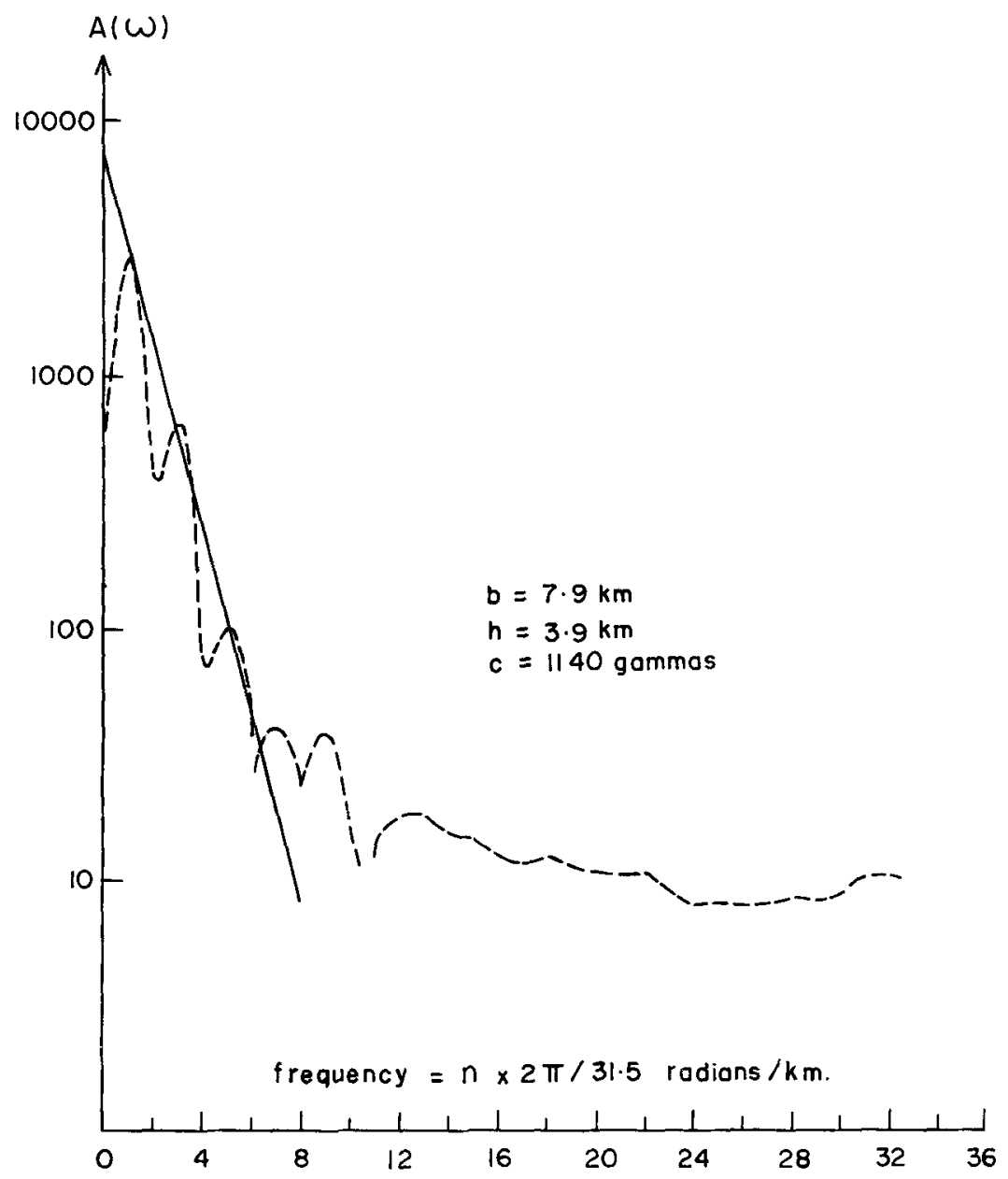

Figure 5. Fourier amplitude $[A(w)]$ spectrum of the magnetic anomaly shown in figure 4a.

interpretation that the depth to the basement in this region is around $2.0 \mathrm{~km}$ below ground surface.

\section{Acknowledgment}

The authors are grateful to Dr D Atchuta Rao for encouragement.

\section{References}

Atchuta Rao D and Rambabu H V 1981 Pure Appl. Geophys. 1191037

Bhimasankaram V L S, Mohan N L and Seshagirirao S V 1978 Geoexploration 16259

Erdelyii 1954 Table of integral transforms (New York: McGraw-Hill) Vol.1 
Gay S P 1963 Geophysics 28161

Koulomzine Th, Lamontagne $Y$ and Nadeau A 1970 Geophysics 35812

Nettleon L L 1976 Gravity and magnetics in oil prospecting (New York: McGraw-Hill)

Rao B S R, Murthy I V R and Rao C V 1973 Geophysics 38710

Rao B S R, Murthy I V R and Rao D B 1978 J. Geophys 44257

Telford W M, Geldert L P, Sheriff R E and Keys D A 1976 Applied geophysics (Cambridge: University Press) 